See Article page XXX.

\section{Commentary: Increased left ventricular outflow tract angulation correlates with increased size of ascending aortic aneurysms and aortic wall shear stress: But which comes first?}

\section{Nicholas T. Kouchoukos, MD}

In this issue of the Journal, Salmasi and colleagues ${ }^{1}$ examine the relationship between the geometry (angulation) of the ventriculoaortic junction and blood flow patterns in 33 patients with ascending thoracic aortic aneurysms who were scheduled for open repair. Patients with connective tissue disorders, bicuspid valves, or aortic stenosis were excluded. The angle created by the left ventricular outflow tract (LVOT), the aortic valve orifice, and the mid-ascending aorta was determined using magnetic resonance imaging. Computational fluid dynamics modeling was performed in 11 of the 33 patients, and several derived parameters representing wall shear stress (WSS) were examined. The principal finding of the study was a strongly positive correlation between the LVOT-aortic angle and the aortic diameter at both the level of the aortic sinus and the ascending aorta. An additional finding was a strong relationship between the LVOT-aortic angle and WSS on the outer curve of the ascending aorta, suggesting that more angulated aortas are subject to greater levels of WSS at this location. This relationship did not exist for other regions of the ascending aorta.

The authors' findings are supported by a recent study by Kauhanen and colleagues, ${ }^{2}$ who measured the angle

\footnotetext{
From the Division of Cardiovascular and Thoracic Surgery, Missouri Baptist Medical Center, BJC Healthcare, St Louis, Mo.

Disclosures: The author reported no conflicts of interest.

The Journal policy requires editors and reviewers to disclose conflicts of interest and to decline handling or reviewing manuscripts for which they may have a conflict of interest. The editors and reviewers of this article have no conflicts of interest.

Received for publication June 19, 2021; revisions received June 19, 2021; accepted for publication June 21, 2021.

Address for reprints: Nicholas T. Kouchoukos, MD, Missouri Baptist Medical Center, 3023 N Ballas Rd, Suite 150D, St Louis, MO 63131 (E-mail: ntkouch@aol.com). J Thorac Cardiovasc Surg 2021; $\mathbf{\square}: 1-2$ $0022-5223 / \$ 36.00$

Copyright (c) 2021 by The American Association for Thoracic Surgery https://doi.org/10.1016/j.jtcvs.2021.06.034
}

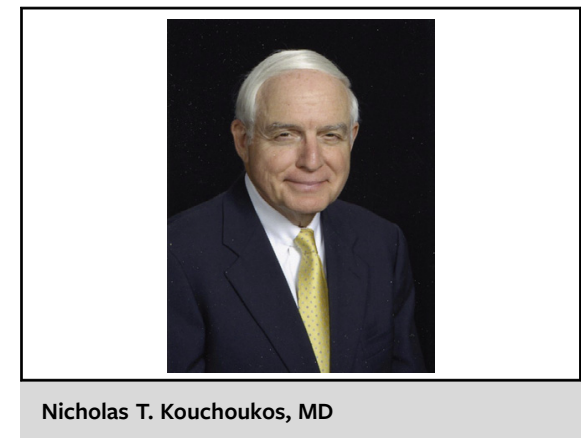

\begin{abstract}
CENTRAL MESSAGE
Increased angulation between the left ventricular outflow tract and the aneurysmal ascending aorta correlates with increased aortic diameter and wall shear stress on the outer curve of the aorta.
\end{abstract}

between the long axis of the heart and the ascending aorta using computed tomography angiography and found a correlation between this angle and the size of the ascending aorta in 28 patients with ascending aortas greater than $40 \mathrm{~mm}$ in diameter. Using 4-dimensional flow magnetic resonance imaging in these 28 patients, they observed that a smaller heart-aorta angle correlated with increased total wall shear stress in the outer curvature of the proximal ascending aorta.

Salmasi and colleagues ${ }^{1}$ postulate that angulated flow in the proximal ascending aorta can lead to jet impingement on its outer curvature and greater WSS as well as turbulent flow in this area, which may accelerate aortic enlargement and aneurysm formation. Unfortunately, tissue samples from the replaced aortas were not examined, and thus whether a correlation, if any, exists, between the calculated WSS and the severity of degenerative changes in the aortic wall could not be determined. A further unanswered question is whether, in the absence of abnormalities of the aortic valve, the angulation develops spontaneously and leads to increased wall stress and aortic enlargement or conversely, whether the degenerative changes that occur in the proximal ascending aorta leading to enlargement increase the degree of angulation. The authors acknowledge that the cause-effect relationship between the abnormal geometry and aneurysm formation cannot be firmly 
established without longitudinal studies involving serial imaging.

A long-term objective of this and other studies examining the relationship between increased WSS and degenerative changes in the wall of the proximal aorta is to identify patients at substantial risk for aortic dissection or rupture before they meet the established size criteria for elective aortic replacement. This is a highly desirable objective, but achieving it will require studies with larger numbers of patients, including those with normal sized ascending aortas, connective tissue disorders, and abnormalities of the aortic valve.

\section{References}

1. Salmasi SF, Pirola S, Mahuttanatan S, Fisichella SM, Sengupta S, Jarral OA, et al. Geometry and flow in ascending aortic aneurysms are influenced by left ventricular outflow tract orientation. J Thorac Cardiovasc Surg. XXX, 2021 [Epub ahead of print].

2. Kauhanen SP, Liimatainhen T, Kariniemi E, Korhonen M, Parkkonen J, Vienonen J, et al. A smaller heart-aorta-angle associates with ascending aortic dilatation and increases wall shear stress. Eur Radiol. 2020;30:5149-57. 